\title{
Metabolomic approaches for phospholipid analysis: advances and challenges
}

\author{
Raúl González-Domínguez*,1 \\ 1 Department of Chemistry, Faculty of Experimental Sciences, International Campus of Excellence (CeiA3), University of Huelva, \\ Huelva 21007, Spain \\ *Author for correspondence: raul.gonzalez@dqcm.uhu.es
}

First draft submitted: 14 April 2018; Accepted for publication: 23 April 2018; Published online: 10 July 2018

Keywords: annotation $\bullet$ biomarkers $\bullet$ mass spectrometry $\bullet$ metabolomics $\bullet$ phospholipids

Phospholipids (PLs) play numerous central roles in biological systems, including the formation of the lipid bilayer in cellular membranes and the regulation of multiple biological pathways by means of liberation of a myriad of signaling molecules, named lysophospholipids, platelet activating factors, eicosanoids and diacylglycerides, which are involved in the modulation of cell proliferation, inflammation, oxidative stress, neurotransmission and many other processes. Thus, alterations in the homeostasis of PLs have major consequences on the organism, therefore they have been previously proposed as potential biomarkers for the investigation of pathogenic hallmarks associated with diseases such as Alzheimer's disease [1] or cancer [2]. In this context, the use of metabolomic approaches has gained great importance in recent years, as they allow us to obtain a comprehensive overview of phospolipidome by the means of simultaneous analysis of the entire set of individual PL species found in a given biological sample. For this purpose, various complementary analytical platforms can be employed, each one having their own inherent strengths and weaknesses [3].

\section{Metabolomic platforms for phospholipid analysis}

Mass spectrometry (MS) is the most commonly employed technique to analyze PLs, for which different instrumental configurations are available. Direct MS analysis, based on direct infusion of samples into the MS system without a previous chromatographic or electrophoretic step, is the simplest MS-based metabolomic approach, which has demonstrated a great utility for high-throughput screening analysis due to its reduced runtime and wide metabolite coverage [4]. In this line, it should also be noted that the multidimensional MS-based shotgun lipidomic (MDMSSL) platform developed by Han et al., which allows direct fingerprinting of various lipid classes through selective ionization and subsequent detection by means of neutral loss or precursor-ion scanning of each lipid class to obtain multidimensional mass spectra [5]. However, these high-throughput lipidomic strategies are usually affected by increased matrix effects due to the direct introduction of the whole sample into the mass spectrometer, and present significant limitations to distinguish between isomeric compounds. Thus, the coupling of liquid chromatography with MS (LC-MS) has been established as the gold standard for the analysis of PLs [6]. Although, normal phase chromatography has been traditionally employed to fractionate major lipid classes, the separation of individual PL species usually requires the application of reversed phase columns. High resolution MS is normally applied to acquire metabolomic fingerprints, but some authors have also demonstrated the potential of tandem MS to detect PLs in a selective and quantitative manner [7]. Complementarily, atomic MS (i.e., inductively coupled plasma MS, ICP-MS) can also be used for the analysis of PLs, additionally enabling their sensitive and selective quantifications without the need of pure standards [8].

Despite all these analytical advances developed for metabolomic fingerprinting of PLs, there are still some important challenges to be addressed. First, it should be noted that the great complexity of the phospholipidome precludes the complete chromatographic resolution of the entire set of PL species present in biological samples. In this sense, the combination of complementary metabolomic platforms has emerged as the most suitable strategy in metabolomic research [3]. Furthermore, PLs are well known to strongly contribute to matrix effects, thus exerting ion suppression over other analytes [9]. This finally results in reduced metabolome coverage, mainly by hindering the 
detection of minor compounds. However, one of the major challenges in current metabolomic research is related to the annotation of discriminant features identified after raw data statistical analysis.

\section{Annotation of PLs}

After MS-based analysis and data processing, the identification of discriminant features is a major bottleneck in the metabolomic pipeline. The Metabolomics Standards Initiative (MSI) defines different levels of metabolite annotation on the basis of several guidelines [10]. Thereby, level I identification requires the analysis of matched standards using the same analytical method. However, this is often impossible due to the unavailability of pure standards, especially for PLs and other lipid classes that are composed by a myriad of species and isomers. On the other hand, metabolite annotation can also be carried out by comparing the experimental accurate masses and MS/MS spectra with those available in MS libraries and published literature (level II).

Particularly, it is noteworthy that PLs show characteristic fragmentation patterns that facilitate their annotation [11-13]. Upon electrospray ionization (ESI), phosphatidylcholines usually yield protonated $\left([\mathrm{M}+\mathrm{H}]^{+}\right)$, sodiated $\left([\mathrm{M}+\mathrm{Na}]^{+}\right)$or potassiated $\left([\mathrm{M}+\mathrm{K}]^{+}\right)$adducts in the positive ion mode, while demethylated ions $\left([\mathrm{M}-\mathrm{CH} 3]^{-}\right)$, chloride adducts $\left([\mathrm{M}+\mathrm{Cl}]^{-}\right)$or formate adducts $\left([\mathrm{M}+\mathrm{FA}]^{-}\right)$are usually generated under negative ionization. For ethanolamine, serine and glycerol species, major metabolic features usually observed are $[\mathrm{M}+\mathrm{H}]^{+}$and $[\mathrm{M}-\mathrm{H}]^{-}$, in positive and negative modes respectively; while phosphatidylinositols and phosphatidic acids are mainly detected in the form of $[\mathrm{M}-\mathrm{H}]^{-}$adducts under negative ionization. MS/MS spectra of phosphatidylcholines is dominated by characteristic daughter ions at $\mathrm{m} / \mathrm{z} 184,104$ and $86 \mathrm{Da}$ in positive ionization mode, and two neutral losses due to the elimination of the trimethylamine and the phosphocholine head group. On the other hand, product-ion scanning of ethanolamines, serines and glycerolsnormally yields $[\mathrm{M}+\mathrm{H}-141]^{+},[\mathrm{M}+\mathrm{H}-185]^{+}$and $[\mathrm{M}+\mathrm{H}-172]^{+}$ fragments, coming from the release of the phosphoethanolamine, phosphoserine and phosphoglycerol moieties, respectively. In the negative ion mode, these typical fragments are observed at $\mathrm{m} / \mathrm{z} 153,168,196,241,171$ and $[\mathrm{M}-\mathrm{H}-87]^{-}$, for phosphatidic acids, phosphocholines, phosphoethanolamines, phosphoinositols, phosphoglycerols and phosphoserines, respectively [11]. Furthermore, tandem MS also enables the annotation of individual PL species thanks to characteristic ions generated in the positive ion mode through the breakage of ester bonds between fatty acids and the glycerol backbone, or the study of carboxylate anions released under negative ionization [8,12].

\section{Conclusion}

Metabolomic fingerprinting of PLs has a great potential in biomedical research because of the pivotal role of these lipids in health and pathogenesis of numerous diseases. For this purpose, direct MS-based metabolomic strategies can be used to carry out a preliminary screening in a simple and rapid manner. However, the application of hyphenated LC-MS platforms is best suited for obtaining wider metabolome coverage. Anyway, considering the great chemical complexity of the lipidome, the combination of complementary metabolomic platforms stands out as the most reliable alternative. On the other hand, the great challenge that metabolite annotation entails within the metabolomic workflow should also be noted. In the particular case of PLs, characteristic MS spectra and fragmentation patterns are obtained upon ESI-MS analysis depending on the phospholipid class and fatty acid composition, thus facilitating their annotation.

As a consequence of these technical advances in the field of metabolomic fingerprinting and subsequent annotation of PLs, numerous authors have previously demonstrated the involvement of abnormal phospholipid homeostasis in the development of multiple and heterogeneous diseases, including cancer, Alzheimer's disease, diabetes and other disorders $[1,2,4]$. However, the implementation of these 'putative biomarkers' in the clinical practice requires the application of confident validation studies, which are usually missed in most metabolomic studies. To this end, the use of targeted metabolomic techniques is very interesting since the specific quantification of metabolites is allowed, normally with increased sensitivity compared with that provided by untargeted approaches.

Financial \& competing interests disclosure

The author has no relevant affiliations or financial involvement with any organization or entity with a financial interest in or financial conflict with the subject matter or materials discussed in the manuscript. This includes employment, consultancies, honoraria, stock ownership or options, expert testimony, grants or patents received or pending, or royalties.

No writing assistance was utilized in the production of this manuscript. 


\section{References}

1. Kosicek M, Hecimovic S. Phospholipids and Alzheimer's disease: alterations, mechanisms and potential biomarkers. Int. J. Mol. Sci. 14(1), 1310-1322 (2013).

2. Bandu R, Mok HJ, Kim KP. Phospholipids as cancer biomarkers: mass spectrometry-based analysis. Mass Spectrom. Rev. 37(2), 107-138 (2018).

3. González-Domínguez Á, Durán-Guerrero E, Fernández-Recamales Á et al. An overview on the importance of combining complementary analytical platforms in metabolomic research. Curr. Top. Med. Chem. 17(30), 3289-3295 (2017).

4. González-Domínguez R, Sayago A, Fernández-Recamales Á. Direct infusion mass spectrometry for metabolomicphenotyping of diseases. Bioanalysis 9(1), 131-148 (2017).

5. Han X, Yang J, Cheng H, Ye H, Gross RW. Toward fingerprinting cellular lipidomes directly from biological samples by two-dimensional electrospray ionization mass spectrometry. Anal. Biochem. 330(2), 317-331 (2004).

6. Brouwers JF. Liquid chromatographic-mass spectrometric analysis of phospholipids. Chromatography, ionization and quantification. Biochim. Biophys. Acta. Mol. Cell Biol. Lipids. 1811(11), 763-775 (2011).

7. Cui Z, Thomas MJ. Phospholipid profiling by tandem mass spectrometry. J. Chromatogr. B Analyt. Technol. Biomed. Life Sci. 877(26), 2709-2715 (2009).

8. González-Domínguez R, García-Barrera T, Gómez-Ariza JL. Combination of metabolomic and phospholipid-profiling approaches for the study of Alzheimer's disease. J. Proteomics 104, 37-47 (2014).

9. As PL. Phospholipid-based matrix effects in LC-MS bioanalysis. Bioanalysis 3(4), 349-352 (2011).

10. Sumner LW, Amberg ÆA, Barrett ÆD et al. Proposed minimum reporting standards for chemical analysis: Chemical Analysis Working Group (CAWG) Metabolomics Standards Initiative (MSI). Metabolomics 3(2), 211-221 (2007).

11. Pulfer M, Murphy RC. Electrospray mass spectrometry of phospholipids. Mass Spectrom. Rev. 22(5), 332-364 (2003).

12. Wang C, Xie S, Yang J, Yang Q, Xu G. Structural identification of human blood phospholipids using liquid chromatography/quadrupole-linear ion trap mass spectrometry. Anal. Chim. Acta 525(1), 1-10 (2004).

13. González-Domínguez R, González-Domínguez Á, Sayago A, Fernández-Recamales Á. Mass spectrometry-based metabolomic multiplatform for Alzheimer's disease research. In: Biomarkers for Alzheimer's Disease Drug Development. Robert Perneczky (Ed.). Humana Press (Springer), NY, USA, 125-137 (2018). 
